\title{
Effect of Addiction of Diacetylmorphine on Sperm Ultrastructural Morphology \\ http://bjas.journals.ekb.eg
}

A.A.Ebrahim ${ }^{1}$, H.H.Sabry ${ }^{1}$, D.M.Ali ${ }^{1}$, A.A.El Fallah ${ }^{2}$ and D.G.Yossef ${ }^{1}$

${ }^{1}$ Dermatology; Andrology and Venereology Dept., Faculty of Medicine, Benha Univ., Benha, Egypt

${ }^{2}$ Clinical and Chemical Pathology Dept., Faculty of Medicine, Benha Univ., Benha, Egypt

E-Mail:ahmed2356@gmail.com

\begin{abstract}
Addiction is a major problem confronting the whole world today. Disruption of interpersonal relationships, economic loss, and crimes against property are frequent consequences. Harm to the individual himself extends to all physiological systems, Aim and objectives: The aim of this study is to evaluate the effect of diacetylmorphine addiction on sperm ultrastructral morphology by electron microscope in patient attending Benha Psychiatric Hospital, Subjects and methods: An observational cross-sectional study was done including 50 addict patients who were admitted in addiction section in Benha psychiatry hospital. Patient who were addict on diacetylmorphine; age> 20 years old for at least one year were enrolled in this study. The duration of the study ranges from 6 to 12 months, Results: The present study shows that Acrosome present was 84.1 \pm 12.4 , Nucleus Normal shape was $46.1 \pm 6.8$, Nucleus Normal shape was $46.1 \pm 6.8$,Chromatin was $70.1 \pm 1.7$,Cytoplasmic residue absent was $74.6 \pm$ 5.7,Mitochondria Normal shape was $85.2 \pm 8.5$,Normal helix was $83.1 \pm 4.3$, Axoneme Normal pattern was $56.7 \pm 8.4$,Normal arms was $57.3 \pm 6.8$,Normal shape was $90.4 \pm 4.1$, Accessory fiber normal was $77.1 \pm 11.6$ ,Fibrous sheath normal $99.7 \pm 15.3$ Plasma membrane normal was $90.2 \pm 12.3$, Conclusion: Heroin consumption affects sperm maturities such as histone-to-protamine ratio and impair semen profile in general and particularly sperm morphology and motility. Heroin may be considered as one of the idiopathic male infertility reasons.
\end{abstract}

Keywords: Observational, Addiction, Infertility, Sperm, and Ultrastructral.

\section{Introduction}

Infertility is defined as the failure to achieve a successful pregnancy after 12 months or more of regular unprotected intercourse, and male-factor infertility is the cause in $50 \%$ of all infertile couples [1].

Several conditions may explain male-factor infertility such as varicocele, cryptorchidism, infections, obstructive lesions, cystic fibrosis, trauma, tumors,oxidativestress, and drugs which can lead to sperm damage, deformity and eventually male infertility [2].

Semen analysis is routinely used to evaluate the male partner in infertile couples. Measurement of sperm concentration, motility, and morphology all provide useful information for diagnosing male infertility [3].

Sperm morphology, as measured according to strict criteria, appears to be the most informative semen measurement for discriminating between fertile and infertile men. However, none of the measures, alone or in combination, can be considered diagnostic of infertility [2].

Drug addiction can be an important cause of male factor infertility and includes use of anabolicandrogenic steroids, marijuana, opioid narcotics, cocaine, and methamphetamines [4].

Diacetylmorphine or diamorphine (known in population as heroin) is an opioidwhich acts like morphine in the body. Heroin isasemi-synthetic opioid, meaning that it was created from an opiate that occurs in nature (morphine). Heroin is a white or brown powder made from the sap of the poppyplant. It is an analgesicdrug (painkiller), and its effects are like other drugs that come from the poppy plant sap, like opiumand morphine [4].
Diacetylmorphine act on the hypothalamicpituitary axis by inhibiting the pulsatility of GnRH secretion: the resulting suppression of FSH and LH release consequently leads to impaired spermatogenesis and reduced testosterone concentrations [5].

Effects of opioids on endocrine parameters was studied and concluded that there is still insufficient information on the long-term effects of opioids in regards to fertility despite concrete evidence of opioid-induced hypogonadism [6].

Transmission electron microscopy (TEM) represents a valuable method to explore the in vitro effects of different compounds (for example drugs with potential spermicidal activity) on theultrastructural morphology of human spermatozoa [7].

Use our understanding of the structure of the human spermatozoon and has of thetransmisionelectron microscope has greatly expanded enabled us to define more precisely the nature of the defect in different types of infertility [7].

The aim of this study is to evaluate the effect of diacetylmorphine addiction on sperm ultrastructral morphology by electron microscope in patient attending Benha Psychiatric Hospital.

\section{Patients and methods}

This was observational cross-sectional study, which had been conducted on patients who were addict diacetylmorphine. All patients had been recruited from addiction section in Benha Psychiatry Hospital.

An approval from the Research Ethics Committee in Benha Faculty of Medicine had been obtain to conduct this study .A written Informed consent will be obtained from all participants. It included all 
details about the stud $\mathrm{y}($ title,objectives,methods, expected benefits and confidentially of data).

The study had been conducted on addict patients who are admitted in addiction section in Benha psychiatry hospital. The least number of patients (50 participants) had been included in the study.

\section{- Inclusion criteria}

Patient who were addict on diacetylmorphine, age> 20 years old for at least one year had been enrolled in this study.

\section{- Exclusion criteria}

Patients who were suffering from clinical causes that may affects spermatogenesis such as diabetes, hypertension, cardiac diseases and varicocele will be excluded from this study.

Each patient will be subjected to the following:

1- Psychiatric consultation: by a psychiatrist using Diagnostic and Statistical Manual of Mental Disorders (DMS IV) Criteria that describes the symptoms for all mental disorders.

2- Full history taking: including items about sociodermographic data: age, sex, origin, residence, marital status, smoking, special habits, education, occupation, social class and history of drugs intake.

3- General clinical examinations: to exclude systemic diseases.
4- Local examination to detect varicocele, hydrocele and any congenital

5- Laboratory investigations; Collect semen sample in astrile container from diacetylmorphine addict patient.

\subsection{Statistical analysis}

Data collected throughout history, basic clinical examination, laboratory investigations and outcome measures coded, entered and analyzed using Microsoft Excel software. Data were then imported into Statistical Package for the Social Sciences (SPSS version 20.0) (Statistical Package for the Social Sciences) software for analysis. According to the type of data qualitative represent as number and percentage, quantitative continues group represent by mean $\pm \mathrm{SD}$, The other continuous variables and ordinal variables were presented by the median and 25th and 75th percentiles. Number and percentage were reported for categorical variables. The following tests were used to test differences for significance;. Difference and association of qualitative variable by Chi square test $\left(\mathrm{X}^{2}\right)$. Differences between quantitative independent groups by $\mathrm{t}$ test, multiple by ANOVA, correlation by Pearson's correlation agreement by Kappa . P value was set at $<0.05$ for significant results $\&<0.001$ for high significant result.

\section{Results}

The demographic data of included patients sgown in Table (1).

Table (1) Demographic characteristics between studied groups.

\begin{tabular}{llcccc}
\hline Variables & Addicted $(\mathbf{n = 5 0})$ & $\begin{array}{c}\text { Control } \\
(\mathbf{n = 5 0})\end{array}$ & t & P \\
\hline $\begin{array}{l}\text { Age (years) } \\
\text { Mean } \pm \text { SD }\end{array}$ & & $44.14 \pm 6.45$ & $43.57 \pm 6.82$ & .553 & .581 \\
Residence & Urban & $35(70 \%)$ & $32(64 \%)$ & .407 & .523 \\
& Rural & $15(30 \%)$ & $18(36 \%)$ & & .421 \\
Social class & Middle & $30(60 \%)$ & $26(52 \%)$ & .649 & \\
& High & $20(40 \%)$ & $24(48 \%)$ & & \\
\hline
\end{tabular}

There is a significant difference between the two groups regarding smoking, tobacco consumption and history of drugs intake. Thus, all patients were smokers, while only $32 \%$ of the control were smoker, mean of duration of dependence (year) was 12.4 \pm 4.1 , mean of Duration of Heroin consumption (year) was 8.4.4 \pm 1.1 and Heroin use was $1.1 \pm 0.02$ (mg/day) Table (2).

Table (2) Lifestyle factors and Heroin consumption data among studied patients.

\begin{tabular}{|c|c|c|c|c|c|c|}
\hline \multirow[t]{2}{*}{ Variables } & \multicolumn{2}{|c|}{$\begin{array}{l}\text { Addicted } \\
(\mathbf{n}=50)\end{array}$} & \multicolumn{2}{|c|}{$\begin{array}{c}\text { Control } \\
(\mathbf{n}=\mathbf{5 0})\end{array}$} & \multirow[t]{2}{*}{$\chi^{2} / \mathrm{t}$} & \multirow[t]{2}{*}{$\mathbf{P}$} \\
\hline & $\mathbf{N}$ & $\%$ & $\mathbf{N}$ & $\%$ & & \\
\hline Smoking & 50 & 100 & 16 & 32 & 51.5 & .000 \\
\hline Tobacco (cigarettes/day) & \multicolumn{2}{|c|}{$13.1 \pm 5.2$} & \multicolumn{2}{|c|}{$6.98 \pm 5.54$} & 5.63 & .001 \\
\hline \multicolumn{7}{|l|}{ Mean \pm SD } \\
\hline History of drugs intake & 50 & 100 & 4 & 8 & 54.9 & .000 \\
\hline $\begin{array}{l}\text { Dependence Duration (year) } \\
\text { Mean } \pm \text { SD }\end{array}$ & \multicolumn{2}{|c|}{$8.2 \pm 5.3$} & \multicolumn{2}{|c|}{---- } & -- & -- \\
\hline
\end{tabular}


Table (2) Continue

\begin{tabular}{lrlllll}
\hline Heroin use (mg/day) & & \multicolumn{2}{c}{$1.26 \pm 0.2$} & ---- & -- & -- \\
$\begin{array}{l}\text { Mean } \pm \text { SD } \\
\text { Other }\end{array}$ & Heroin alone & 13 & 26 & & --- & -- \\
addiction & Marijuana & 28 & 56 & ---- & -- & -- \\
& Tramadol & 29 & 58 & ---- & -- & -- \\
\hline
\end{tabular}

There is a significant difference between the two groups regarding sexual intercourse frequency per month. Fig (1).

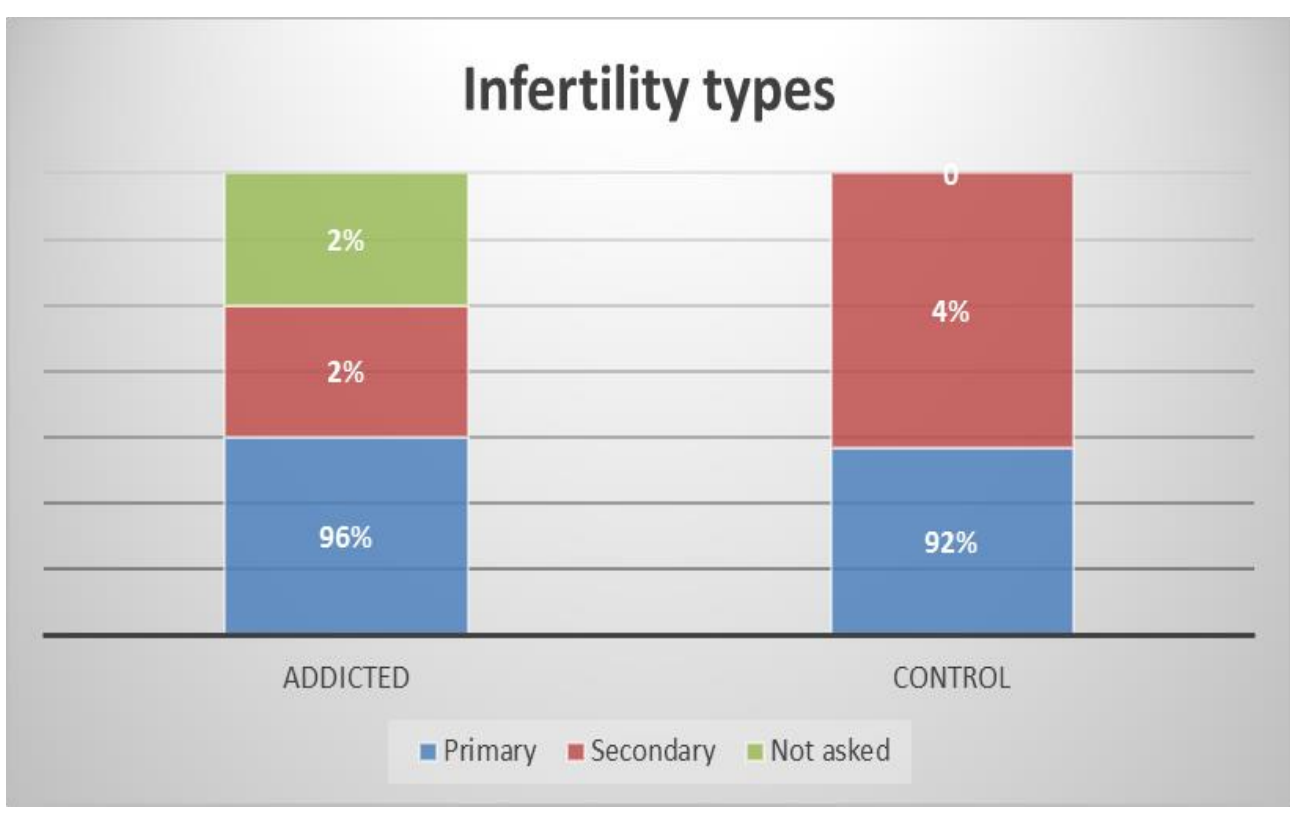

Fig (1) Type of infertility among studied patients.

There a significant difference between the two groups regarding FSH, LH and testosterone Fig (2).

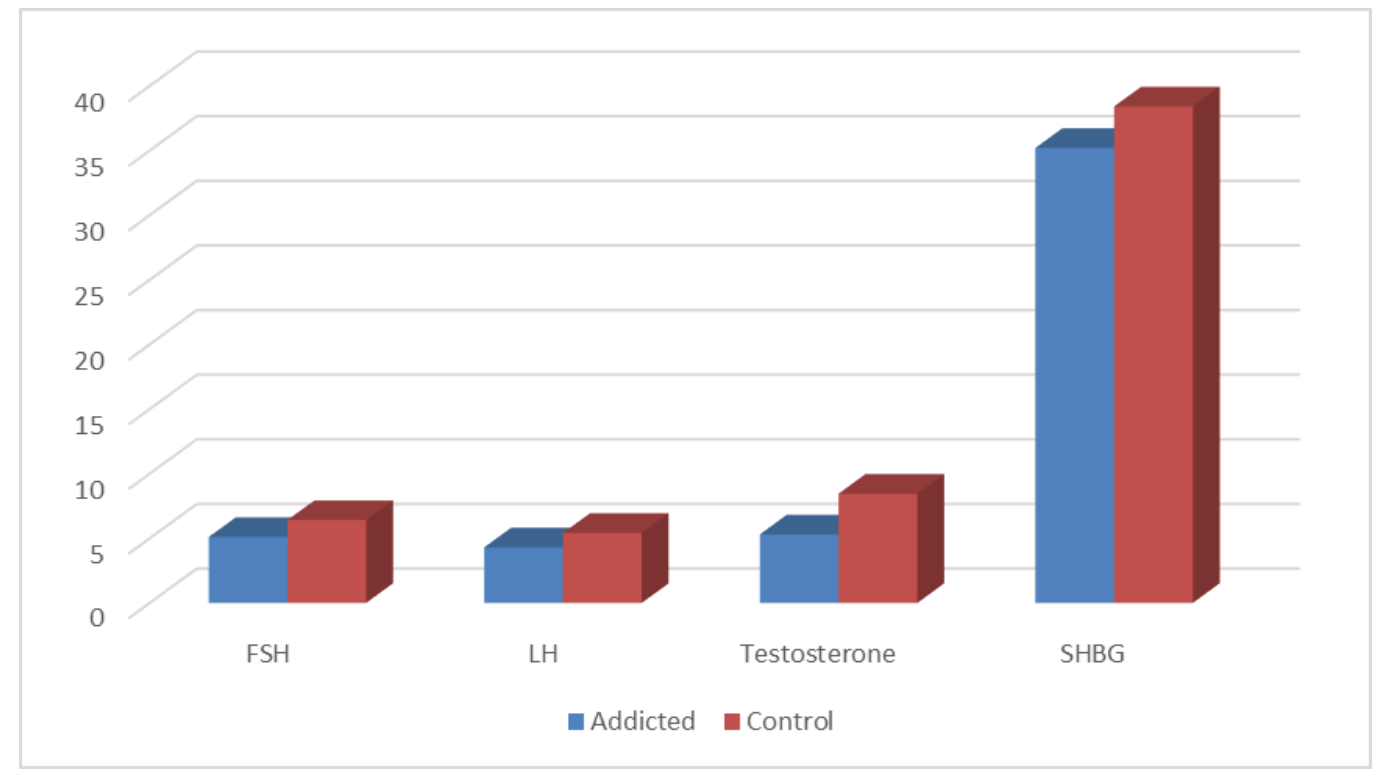

Fig (2) Plasma hormone levels in the study group

There is a significant difference between the groups regarding sexual abstinence, semen $\mathrm{pH}$ and concentration, sperm progressive motility, semen vitality and sperm normal forms Table (3). 
Table (3) Sperm baseline parameters in of infertile males.

\begin{tabular}{|c|c|c|c|c|c|}
\hline Variables & & $\begin{array}{c}\text { Addicted } \\
(n=50)\end{array}$ & $\begin{array}{c}\text { Control } \\
(n=50)\end{array}$ & Test & $\mathbf{P}$ \\
\hline \multirow[t]{8}{*}{ Physical parameters } & $\mathrm{pH}$ & $7.1 \pm 0.98$ & $7.9 \pm 0.8$ & 5.48 & 0.02 \\
\hline & Mean \pm SD & & & & \\
\hline & Volume (mL) & $2.785 \pm 2.1$ & $4.03 \pm 1.49$ & 1.91 & 0.049 \\
\hline & Mean \pm SD & & & & \\
\hline & Concentration $\left(\times 10^{6} / \mathrm{mL}\right)$ & $163.1 \pm 38.4$ & $168.3 \pm 34.22$ & 0.46 & 0.56 \\
\hline & Mean \pm SD & & & & \\
\hline & Viability (\%) & $72.54 \pm 3.4$ & $87.53 \pm 2.06$ & 4.69 & 0.01 \\
\hline & Mean \pm SD & & & & \\
\hline \multirow[t]{2}{*}{ Sperm motility } & total & $41.9 \pm 1.8$ & $68.56 \pm 2.2$ & 4.61 & 0.001 \\
\hline & progressive Mean \pm SD & $22.16 \pm 3.2$ & $38.32 \pm 4.47$ & 5.12 & 0.001 \\
\hline Abnormal & Head & $73.93 \pm 3.79$ & $4.17 \pm 1.08$ & 7.32 & 0.001 \\
\hline \multirow[t]{3}{*}{ morphology } & Tail & $13.83 \pm 2.42$ & $3.8 \pm 0.96$ & 3.54 & 0.031 \\
\hline & total & $87.76 \pm 3.43$ & $7.97 \pm 1.02$ & 7.44 & 0.001 \\
\hline & Histone-to-protamine ratios & $6.67 \pm 1.13$ & $32.65 \pm 9.13$ & 2.61 & 0.04 \\
\hline
\end{tabular}

\section{Discussion}

The current study shows that, mean of infertility duration (months) was $41.9 \pm 14.1$, mean of duration of heroin consumption (year) was 8.4.4 \pm 1.1 , most common type of infertility was primary (96\%) . Mean Sexual intercourse frequency per month was $10.1 \pm 2.4$.

Plasma hormone levels, mean of FSH was $5.1 \pm$ $2.9(\mathrm{mUI} / \mathrm{mL}), \mathrm{LH}(\mathrm{mUI} / \mathrm{mL}) \quad$ was $4.3 \pm 2.1$, mean of testosterone was $5.3 \pm 2.2$, mean SHBG: Sex hormone binding globulin was $35.2 \pm$ 15.6 .

Our results are supported by study of Nazmara et al., [8] as they reported that serum sex hormone levels were not significantly differed between groups (Heroin Addict and healthy). There was a correlation between the amount of daily heroin consumption and LH level.

As regard Gundersen et al., [9], cannabis smokers have been shown to have higher levels of testosterone than cannabis non-smokers. However, the plasma testosterone levels were within the same range as those observed in cigarette smokers.

In the study in our hands, semen volume and its viscosity, agglutination, aggregation, sperm concentration. Semen $\mathrm{pH}(7.9 \pm 0.6)$ and sperm volume and Concentration $(\times 106 / \mathrm{mL}) \quad(3.42 \pm$ $1.7 v s 24.1 \pm 8.4$ respectively). Total sperm number $(\times 106 /$ jaculate $)$ was $77.6 \pm 55.4$, Sperm progressive motility $(\mathrm{a}+\mathrm{b}, \%)$ was $33.5 \pm 16.2$, Vitality (\%) was $28.4 \pm 15.1$. Sperm normal forms $(\%)$ was $1.51 \pm$ 0.21 . As regarding multiple anomaly indexes, $46 \%$ was Oligozoospermia,72\% of them asthenozoospermia and $60 \%$ were Teratozoospermia.

Our results are in line with study of Verhaeghe et al., [10] as they reported that except for sexual abstinence, which was significantly lower in the group of cannabis users, the conventional semen parameters did not vary significantly between the two groups. Leucocytospermia was not detected in the semen samples of cannabis users and cannabis nonusers. Oligozoospermia was observed in $44 \%$ (12 of 27 ) of the cannabis users and $41 \%$ (11 of 27) of the cannabis non-users. Asthenozoospermia was present in $74 \%$ (20 of 27) of the cannabis users and 63\% (17 of 27) of the cannabis non-users. Teratozoospermia was observed in 59\% (16 of 27) of the cannabis users and $48 \%$ (13 of 27) of the cannabis non-users.

Furthermore, Gundersen et al., [9] observed that men using cannabis more than once per week have significantly lower sperm concentrations and total sperm counts than non-users, and these impairments are worsened by the use of additional recreational drugs (). $\triangle 9$-THC induces a concentration-dependent decrease in sperm motility and a marked decrease in the percentage of spermatozoa undergoing the spontaneous acrosome reaction [11].

A meta-analysis found that smoking was associated with a $13-17 \%$ decrease in sperm concentration [12].

Data from the study of Joo et al., [13] indicated an association between smoking and sperm count, but not semen volume, motility or morphology. Alcohol consumption was associated with an increase in morphologically abnormal sperm in the present study.

According to Nazmara et al., [8], semen pH (7.8 vs. 7.75$)$, sperm motility $(42.93 \pm 3.89 \%$ vs. $68.9 \pm$ $2.68 \%)$, and viability $(73.27 \pm 3.85 \%$ vs. $86.48 \pm$ $1.05 \%)$, and sperm histone replacement abnormalities $(32.33 \pm 10.89 \%$ vs. $5.56 \pm 0.85 \%)$ were significant differences in addicted group vs. non-exposed ones.

The present study shows that Acrosome present was $84.1 \pm 12.4$, Nucleus Normal shape was $46.1 \pm$ 6.8, Nucleus Normal shape was $46.1 \pm 6.8$ ,Chromatin was $70.1 \pm 1.7$,Cytoplasmic residue absent was $74.6 \pm 5.7$,Mitochondria Normal shape was $85.2 \pm 8.5$,Normal helix was $83.1 \pm 4.3$ ,Axoneme Normal pattern was $56.7 \pm 8.4$,Normal arms was $57.3 \pm 6.8$,Normal shape was $90.4 \pm 4.1$ 
,Accessory fibre normal was $77.1 \pm 11.6$,Fibrous sheath normal $99.7 \pm 15.3$ Plasma membrane normal was $90.2 \pm 12.3$.

Our results are supported by study of Verhaeghe et al., [10] as they reported that a significant increase in the rates of XY hyperhaploid and 18 disomic spermatozoa was found in cannabis users compared to these rates in cannabis non-users $(\mathrm{P}=0.0009$ and $\mathrm{P}$ $=0.0121$, respectively). The aneuploid, diploid and total chromosome abnormality rates were higher in the spermatozoa from cannabis users than in the spermatozoa from cannabis non-users $(\mathrm{P}=0.044, \mathrm{P}=$ 0.037 and $\mathrm{P}=0.0027$, respectively). Meiotic nondisjunctions occurred preferentially during meiosis I in both cannabis users and cannabis non-users $(\mathrm{P}=$ 0.0002 and $P=0.0126$, respectively). However, the rate of meiosis I non-disjunctions was significantly higher in cannabis users than in cannabis non-users $(\mathrm{P}=0.0005)$. The rate of sperm DNA fragmentation was significantly higher in cannabis users than in cannabis non-users $(\mathrm{P}=0.027)$. Chromatin condensation defects did not vary significantly between the two groups. Furthermore, the sperm head length, width and area did not differ significantly between cannabis users and cannabis non-users. The sperm head RVA and the percentage of type 3 spermatozoa.

Cannabinoids can consequently interact with the mitotic apparatus responsible for chromosomal segregation errors. $\triangle 9$ - THC treatment is known to induce chromosome segregation errors in human lymphocytes in vitro, with anaphase lags and unequal segregations in bipolar divisions. Furthermore, it has been proposed that $\triangle 9-\mathrm{THC}$, by affecting the formation of microtubules and spindles, may be considered a mitotic poison .In mice, $\triangle 9$-THC can also modify the permeability of the membrane to ions such as calcium, which is a known inhibitor of the microtubule polymerization and disrupts actin microfilament assembly [14].

The higher levels of numerical chromosome abnormalities observed in the spermatozoa of infertile males who were cannabis users may be the consequence of the clastogenic action of the exocannabinoids or the cannabinoid-induced disruption of mitotic/meiotic events or both. $\Delta 9$ THC and AEA are inhibitors of cell proliferation and inducers of apoptosis [15].

In addition, Nazmara et al., [8] reported that heroin can impair semen quality and alter sperm microenvironment by semen acidification and leukocytospermia, which probably affects the structure and function of these surface-expressed enzymes and influences semen parameters.

There were significant differences in the numbers of sperm with morphologically abnormal nuclei and plasma membranes associated with alcohol consumption in the study of Joo et al., [13].

\section{Conclusion}

Heroin consumption affects sperm maturities such as histone-to-protamine ratio and impairs semen profile in general and particularly sperm morphology and motility. Heroin may be considered as one of the idiopathic male infertility reasons.

\section{References}

[1] F. Zegers-Hochschild, "The international committee for monitoring assisted reproductive technology (ICMART) and the world health organization (WHO) revised glossary on ART terminology, 2009," Hum. Reprod, Vol. 24(11), PP.2683-2687, 2009.

[2] A. Agarwal , S. A. Prabakaran, "Mechanism, measurement, and prevention of oxidative stress in male reproductive physiology,", Vol.5(3),pp.20-52,2005.

[3] P. J. Rowe, F. H. Comhaire, T. B. Hargreave, H. J. Mellows, W. H. Organization, WHO manual for the standardized investigation and diagnosis of the infertile couple. Cambridge, Mass.: Cambridge University Press, 1993.

[4] C. M. Fronczak, E. D. Kim, A. B. Barqawi, "The insults of illicit drug use on male fertility," J. Androl, Vol. 33( 4), PP.515-528, 2012.

[5] C. Vuong, S. H. M. Van Uum, L. E. O’Dell, K. Lutfy, T. C. Friedman, "The effects of opioids and opioid analogs on animal and human endocrine systems," Endocr. Rev, Vol. 31( 1), PP.98-132, 2010.

[6] M. R. Safarinejad , "The effects of opiate consumption on serum reproductive hormone levels, sperm parameters, seminal plasma antioxidant capacity and sperm DNA integrity," Reprod. Toxicol, Vol. 36, PP.18-23, 2013.

[7] E. Moretti, G. Sutera, G. Collodel, "The importance of transmission electron microscopy analysis of spermatozoa: Diagnostic applications and basic research," Syst. Biol. Reprod. Med, Vol. 62(3), PP.171-183, 2016.

[8] Z. Nazmara , "The effect of heroin addiction on human sperm parameters, histone-to-protamine transition, and serum sexual hormones levels," Urol. J, Vol. 16( 3), PP.289-294, 2019.

[9] T. D. Gundersen, "Association between use of marijuana and male reproductive hormones and semen quality: a study among 1,215 healthy young men," Am. J. Epidemiol, Vol. 182( 6), PP.473-481, 2015.

[10]F. Verhaeghe , "Cannabis consumption might exert deleterious effects on sperm nuclear quality in infertile men," Reprod. Biomed. Online, Vol. 40(2), PP.270-280, 2020.

[11]L. B. Whan, M. C. L. West, N. McClure, S. E. M. Lewis, "Effects of delta-9tetrahydrocannabinol, the primary psychoactive cannabinoid in marijuana, on human sperm function in vitro," Fertil. Steril, Vol. 85(3), PP.653-660, 2006.

[12]E. Y. Adashi, M. F. Vine, B. H. Margolin, H. I. 
Morrison, B. S. Hulka, "Cigarette smoking and sperm density: a meta-analysis," Fertil. Steril, Vol. 61(1), PP.35-43, 1994.

[13] K. J. Joo, Y. W. Kwon, S. C. Myung, T. H. Kim, "The effects of smoking and alcohol intake on sperm quality: light and transmission electron microscopy findings," J. Int. Med. Res, Vol. 40(6), PP.2327-2335, 2012.

[14] S. Dalterio, R. Steger, J. Peluso, L. De Paolo,
"Acute $\Delta 9$-tetrahydrocannabinol exposure: effects of hypothalamic-pituitary-testicular activity in mice," Pharmacol. Biochem. Behav, Vol. 26(3), PP.533-537, 1987.

[15]M. Maccarrone , A. Finazzi-Agro, "The endocannabinoid system, anandamide and the regulation of mammalian cell apoptosis," Cell Death Differ, Vol. 10(9), PP.946-955, 2003. 